University of Wollongong

Research Online

Australian Institute for Innovative Materials -

Papers

Australian Institute for Innovative Materials

$1-1-2012$

\title{
Microporous gel polymer electrolytes for lithium rechargeable battery application
}

Nurul Hayati Idris

University of Wollongong, Universiti Malaysia Terengganu, nhi423@uow.edu.au

Md. Mokhlesur Rahman

University Of Wollongong

Jia-Zhao Wang

University of Wollongong, jiazhao@uow.edu.au

Hua-Kun Liu

University of Wollongong, hua@uow.edu.au

Follow this and additional works at: https://ro.uow.edu.au/aiimpapers

Part of the Engineering Commons, and the Physical Sciences and Mathematics Commons

Research Online is the open access institutional repository for the University of Wollongong. For further information contact the UOW Library: research-pubs@uow.edu.au 


\title{
Microporous gel polymer electrolytes for lithium rechargeable battery application
}

\begin{abstract}
Microporous poly(vinylidene fluoride)/poly(methyl methacrylate) (PVDF/PMMA) membranes were prepared using the phase-separation method. Then, the membranes were immersed in liquid electrolyte to form polymer electrolytes. The effects of PMMA on the morphology, degree of crystallinity, porosity, and electrolyte uptake of the PVDF membrane were studied. The addition of PMMA increased the pore size, porosity and electrolyte uptake of the PVDF membrane, which in turn increased the ionic conductivity of the polymer electrolyte. The maximum ionic conductivity at room temperature was $1.21 \times 10-3 \mathrm{~S} \mathrm{~cm}-1$ for Sample E70. The polymer electrolyte was investigated, along with lithium iron phosphate (LiFePO4) as cathode for all solid-state lithium-ion rechargeable batteries. The lithium metal/E70/LiFePO4 cell yielded a stable discharge capacity of $133 \mathrm{mAh} \mathrm{g-1}$ after up to 50 cycles at a current density of $8.5 \mathrm{~mA} \mathrm{~g}-1$.
\end{abstract}

\section{Keywords}

microporous, gel, application, polymer, battery, electrolytes, lithium, rechargeable

Disciplines

Engineering | Physical Sciences and Mathematics

\section{Publication Details}

Idris, N. Hayati., Rahman, M., Wang, J. \& Liu, H. Kun. (2012). Microporous gel polymer electrolytes for lithium rechargeable battery application. Journal of Power Sources, 201 (March), 294-300. 


\title{
Microporous gel polymer electrolytes for lithium rechargeable battery application
}

\author{
Nurul Hayati Idris ${ }^{\text {a }}{ }^{*}$, Md. Mokhlesur Rahman ${ }^{\text {a }}$, Jia-Zhao Wang ${ }^{\text {* }}$, Hua-Kun Liu ${ }^{\text {a }}$ \\ ${ }^{a}$ Institute for Superconducting and Electronic Materials, University of Wollongong, \\ Wollongong, NSW 2519, Australia \\ ${ }^{b}$ Department of Physical Sciences, Faculty of Science, Universiti Malaysia Terengganu, \\ 21030 Kuala Terengganu, Malaysia
}

*Corresponding author. E-mail: jiazhao@uow.edu.au; nhi423@uowmail.edu.au Fax: +61 24221 5731, Phone: +612 42981478 


\begin{abstract}
Microporous poly(vinylidene fluoride)/ poly(methyl methacrylate) (PVDF/PMMA) membranes were prepared using the phase-separation method. Then, the membranes were immersed in liquid electrolyte to form polymer electrolytes. The effects of PMMA on the morphology, degree of crystallinity, porosity, and electrolyte uptake of the PVDF membrane were studied. The addition of PMMA increased the pore size, porosity and electrolyte uptake of the PVDF membrane, which in turn increased the ionic conductivity of the polymer electrolyte. The maximum ionic conductivity at room temperature was $1.21 \times 10^{-3} \mathrm{~S} \mathrm{~cm}^{-1}$ for Sample E70. The polymer electrolyte was investigated, along with lithium iron phosphate $\left(\mathrm{LiFePO}_{4}\right)$ as cathode for all solid-state lithium-ion rechargeable batteries. The lithium metal/E70/LiFePO 4 cell yielded a stable discharge capacity of 133 $\mathrm{mAh} \mathrm{g}^{-1}$ after up to 50 cycles at a current density of $8.5 \mathrm{~mA} \mathrm{~g}^{-1}$.
\end{abstract}

Keywords: Phase separation method; Microporous membrane; Polymer electrolyte; Lithium rechargeable battery. 


\section{Introduction}

Polymer electrolyte may generally be defined as a membrane that possesses transport properties comparable to those of common liquid ionic solutions. The development of polymer electrolytes has drawn the attention of many researchers over the last three decades, as they find applications not only in lithium batteries, but also in other electrochemical devices such as supercapacitors, electrochromic devices, etc. [1]. Lithium batteries use liquid electrolytes with organic solvents, which are flammable and easy to ignite on exposure to high temperatures. To solve this safety problem, there is a strong desire to move to non-flammable electrolytes [2]. In the case of the lithium-ion battery, numerous studies have been conducted to improve its safety performance, since scaled-up forms have promising applications in electric vehicles. Still, one of the main challenges is the electrolytes, because they will react with the active electrode materials [3]. As a result, polymer electrolyte is a promising candidate, as these polymer electrolytes have several advantages over their liquid counterparts. The advantages of these electrolytes include no internal shorting, no leakage of electrolytes, and noncombustible reaction products at the electrode surface, in contrast to the liquid electrolytes [4-9]. However, the prerequisites for a polymer electrolyte for lithium-ion batteries are (i) high ionic conductivity at ambient and sub-ambient temperatures, (ii) good mechanical strength, (iii) appreciable transference number, (iv) thermal and electrochemical stability, and (v) better compatibility with electrodes [6-8]. The polymer electrolyte should also allow good cycle lives at low temperatures and must withstand the internal pressure build-up during battery operation. 
Since 1973 [10], many studies on solid polymer electrolytes have been carried out that involve such techniques as blending, co-polymerizing, cross-linking and adding nanofillers to modify the polymer host to increase the ionic conductivity [4, 11-13]. However, the main obstacle is still the ionic conductivity, which is generally below $10^{-3} \mathrm{~S}$ $\mathrm{cm}^{-1}$ and not enough for practical application. As a result, gelled polymer electrolytes (GPEs) were developed $[4,14,15]$. Much recent attention has turned to microporous gel polymer electrolytes, which can be regarded as an intermediate state between typical liquid electrolytes and dry solid polymer electrolytes. Microporous gel polymer electrolytes usually exhibit ionic conductivities ranging from $10^{-4}$ to $10^{-3} \mathrm{~S} \mathrm{~cm}^{-1}$ at room temperature, near to that of commercial liquid electrolytes. In microporous gel polymer electrolytes, the liquid component is trapped in the polymer matrix, thereby preventing leakage of liquid electrolyte. Consequently, the safety of $\mathrm{Li}$-ion batteries is greatly improved [16]. The pore structure of the polymer membrane is the key component and especially important for the ionic conductivity. The larger the amount of trapped liquid electrolyte in the pores, the higher the ionic conductivity of the membrane [17].

Generally, porous membrane materials are prepared by the phase inversion method [18]. Some of the polymer electrolytes are prepared by template imprinting or the sol-gel process [19], but the preparation process for polymer electrolytes is very tedious, and the pores are also too small to observe, so that such polymer membrane materials are generally classified as non-porous or continuous membranes. Nowadays, researchers are very keen to develop microporous polymer membranes, which can be activated as polymer electrolyte by soaking in an electrolyte solution [20]. The Bellcore technique has been widely used to prepared porous polymer membrane based on poly (vinylidene 
fluoride)-co-hexafluoropropene (PVDF-HFP), which showed favorable ionic conductivity $\left(\sim 1 \mathrm{mS} \mathrm{cm}^{-1}\right)$ at room temperature after soaking with liquid electrolyte [21]. However, the extraction process of dibutyl phthalate (DBP) needs large volumes of organic solvents, which increases the production costs, and the removal of DBP is not $100 \%$ efficient [22-24]. On the other hand, Pasquier et al. [25] have shown that the phase-inversion method is a valid method to use in preparing microporous PVDF-HFP co-polymers by casting a polymer solution and evaporating the solvent and nonsolvent. Research and developments in this field have involved a variety of polymer matrices such poly (ethylene oxide) (PEO) [26-28], poly (acrylonitrile) (PAN) [29, 30], poly (vinyl chloride) (PVC) [31, 32], poly (methyl methacrylate) (PMMA) [33, 34], and polyvinylidene difluoride (PVdF) [2, 35]. In particular, much attention has been devoted to increasing the liquid electrolyte content in the polymer matrix by controlling the components and morphology, as the ionic conductivity of the solid polymer electrolyte increases with increasing amount of the liquid electrolyte trapped in the polymer matrix. In addition, several features of the polymer electrolyte, such as porosity, pore size, crystallinity, chain structure, and the degree of polymerization, contribute to the conduction properties of the carriers [36]. However, high porosity usually leads to fading mechanical strength, and it has been shown that the addition of PEO to the PVDF can improve the porosity and pore connectivity for electrolyte uptake [37, 38], although the mechanical strength of PVDF-PEO blends is lower than that of pure PVDF. In addition, blending PAN and PVDF-HFP can increase the mechanical stability and structural rigidity of the porous polymer electrolyte [39]. 
In this study, we report PVDF-PMMA blends for polymer electrolyte prepared via the phase-separation method in a mixture of $\mathrm{N}, \mathrm{N}$-dimethylformamide (DMF) as a solvent and glycerin as a nonsolvent. The effects of PMMA at different ratios on the morphology, porosity, and electrochemical performance have been characterized with the aim of obtaining better microporous gel polymer electrolyte for lithium-ion rechargeable battery application.

\section{Experimental}

\subsection{Preparation of PVDF/PMMA membranes and polymer electrolytes}

The polymer blend membranes were prepared by the phase-separation method. Poly(vinylidene fluoride) (PVDF, Mw $=534,000$, Sigma-Aldrich) and poly(methyl methacrylate) (PMMA, Mw $=350,000$, Sigma-Aldrich) were dried for 24 hours at $100^{\circ} \mathrm{C}$ in vacuum. The required amounts of PVDF and PMMA were dissolved in a mixture of N,N-dimethyl formamide (DMF, > 99.8\%, Sigma-Aldrich) and glycerin (Fluka) (10:1 by volume), and stirred for 3 hours at $80^{\circ} \mathrm{C}$. Then, the solution was cast onto a glass plate and dried at $120^{\circ} \mathrm{C}$ for 24 hours in vacuum. During this process, DMF and glycerin evaporated in turn, and the voids left by the glycerin formed micropores. The polymer electrolytes were obtained by soaking the obtained membranes in $1 \mathrm{M} \mathrm{LiPF}_{6}$ in a mixture of ethylene carbonate (EC) and dimethyl carbonate (DMC; 1:1 by volume, MERCK, Germany). The compositions of the PVDF/PMMA membranes and the corresponding polymer electrolytes that were prepared are listed in Table 1. M0, M30, M50. M70, M90, and M100 designate the membranes with PVDF to PMMA (wt.\%:wt.\%) weight ratios of 
100:0, 70:30, 50:50, 30:70, 10:90, and 0:100, respectively. The corresponding polymer electrolytes are designated as E0, E30, E50, E70, E90, and E100, respectively.

\subsection{Characterization of the membranes and polymer electrolyte}

The morphology of the membranes was observed by scanning electron microscope (SEM; JOEL JEM-3000). Fourier transform infrared (FTIR) spectroscopy was carried out using a Shimadzu FTIR spectrometer, IRPrestige-21 model. Samples were analysed in attenuated total reflectance (ATR) mode using the Pike MIRacle accessory equipped with a germanium crystal (Pike Technology). Differential scanning calorimetry (DSC) measurements were carried out under argon atmosphere using a Mettler-Toledo thermogravimetric analysis/ differential scanning calorimetry (TGA/DSC) $1 \mathrm{Star}^{\mathrm{e}}$ system from 50 to $200{ }^{\circ} \mathrm{C}$ at $10{ }^{\circ} \mathrm{C} \mathrm{min}^{-1}$. Porosity of the membranes was measured as follows: after weighing, the membrane was immersed in 1-butanol for 2 h. Then, the surface of the membrane was dried by filter paper and weighed again. The porosity was calculated using the following equation:

Porosity $(\%)=\frac{w_{t}-w_{o}}{\rho V} \times 100$

where $V$ is the apparent volume of the membrane, $\rho$ is the density of 1-butanol, and $w_{t}$ and $w_{o}$ are the weights of the wet and dry membranes, respectively. The liquid electrolyte uptake of the membranes was measured according to the following procedure: after weighing, the membrane was immersed in liquid electrolyte for $10 \mathrm{~min}$ and weighed again. The electrolyte uptake was calculated as follows:

Electrolyte uptake (\%) $=\frac{w_{t}-w_{o}}{w_{o}} \times 100$ 
where $w_{t}$ and $w_{o}$ are the weights of the wet and dry membranes, respectively. The impedance of the polymer electrolytes was measured using a CHI 660B electrochemical workstation instrument (Shanghai Chenhua Apparatus, China) in the frequency range from $10 \mathrm{mHz}$ to $100 \mathrm{kHz}$. The polymer electrolytes were cut into $1 \mathrm{~cm}^{2}$ sizes and sandwiched between two blocking stainless steel electrodes. The ionic conductivity of the polymer electrolytes was calculated based on the following equation:

$$
\sigma=\frac{t}{R_{b} A}
$$

Here, $\sigma$ is the ionic conductivity, $R_{b}$ is the bulk resistance, and $t$ and $A$ are the thickness and area of the polymer electrolyte, respectively. Impedance measurements were also carried out in the temperature range between 303 and $373 \mathrm{~K}$. The electrochemical stability window of the polymer electrolyte was determined by linear sweep voltammetry using a stainless steel working electrode and lithium foil as the counter electrode at the scanning rate of $1 \mathrm{mV} \mathrm{s}^{-1}$.

\subsection{Electrochemical characterization}

A mixture composed of 80 wt.\% $\mathrm{LiFePO}_{4}$ powder (DLG Battery Co., Ltd., China), 10 wt.\% carbon black, and 10 wt.\% polyvinylidene fluoride (PVDF) were mixed with $N$-methyl-2-pyrrolidone (NMP). The slurry was spread onto aluminum foil substrates and dried at $100{ }^{\circ} \mathrm{C}$ for $24 \mathrm{~h}$ in vacuum. After being cut to a $1 \times 1 \mathrm{~cm}^{2}$ size, the electrode was mounted as the positive electrode versus lithium metal as the counter and reference electrode in CR 2032 coin-type cells. Here, Sample E70 was chosen as the polymer electrolyte. The $\mathrm{Li} / \mathrm{E} 70 / \mathrm{LiFePO}_{4}$ cell was assembled in an argon-filled glove 
box. Charge-discharge testing was performed galvanostatically between 2.5 and $4.2 \mathrm{~V}$ at room temperature on a Land battery tester (Wuhan Land Electronic Co. Ltd.).

\section{Results and discussion}

A comparison of the cross-sectional morphology of the pure PVDF, pure PMMA, and PVDF/PMMA blend films after extraction of glycerin and DMF from the polymer matrix are shown in Fig. 1. The presence of PMMA leads to change in the size of the pores in the membrane. It was found that the pore size of the membrane increases with the addition of PMMA up to 70 wt.\%, and then the pore size starts to decreases. Sample M70 showed the largest diameter pores (about $10 \mu \mathrm{m}$ ), which is enough to trap more liquid electrolyte and provide a path for ion migration. This trend agrees well with the porosity and liquid electrolyte uptake measurements, as shown in Fig. 2. It can be seen that Sample M70 has the highest porosity of $64 \%$ when compared with pure PVDF (Sample M0) and pure PMMA (Sample M100), which have only 24\% and 41\% porosity, respectively. Such large porosity will help the liquid electrolyte to more easily penetrate into Sample M70, with subsequently more liquid electrolyte embedded in the pores of the membrane. For liquid electrolyte uptake measurements, pure PVDF polymer electrolyte (sample E0) reaches saturation with a weight uptake ratio of $15 \%$. With addition of PMMA to the polymer matrix, the liquid electrolyte uptake ratio increases due to the high porosity of the PVDF/PMMA blended films. Sample E70 reaches saturation with a maximum liquid electrolyte uptake ratio of 204\%. Therefore, the addition of PMMA not only controls the porosity, but also is correlated with the liquid electrolyte uptake of the film, resulting in high ionic conductivity of the membrane. 
Fig. 3 shows the ATR-FTIR spectra of Samples M0, M30, M50, M70, and M100. The characteristic peaks observed are common to both pure PVDF and pure PMMA membranes [40-42]. From Fig 3(a), the vibrational band at $1406 \mathrm{~cm}^{-1}$ corresponds to $\mathrm{CH}_{2}$ wagging deformation of pure PVDF membrane. It can be seen that the intensity of this peak decreases with the addition of PMMA and finally disappears, as shown in Fig. 3(d, e). For pure PMMA membrane, a peak reflecting the stretching of the carbonyl $(\mathrm{C}=\mathrm{O})$ group is present at $1730 \mathrm{~cm}^{-1}$, as shown in Fig. 3(e). From the inset in Fig. 3, it can be clearly seen that the intensity of this peak gradually decreases, while the peak is slightly shifted to $1734 \mathrm{~cm}^{-1}$ in the PVDF/PMMA blend membrane. This result suggests that there is a specific interaction between the carbonyl groups of PMMA and the $\mathrm{CH}_{2}$ groups of PVDF, and indicates the formation of a PVDF/PMMA blended membrane [41, 42].

Fig. 4 shows the differential scanning calorimetry (DSC) curves of the pure PVDF, pure PMMA, and polymer blend membranes. Pure PVDF (Sample M0) shows a characteristic endothermic peak at $159.4^{\circ} \mathrm{C}$, corresponding to the melting temperature of the crystalline form, $T_{\mathrm{m}}$. With the addition of PMMA, the melting temperature of the polymer blend membrane is depressed markedly. However, in the rich PMMA concentration (Sample M90), there was no trace of melting behavior of PVDF. Similar results were obtained by Ma et al. [43]. They reported that the decrease in $T_{\mathrm{m}}$ with increasing PMMA concentration could be related to the melting temperature depression of the crystalline components, suggesting that the growth of crystals is affected by the interaction of the components. The integral peak area under the $T_{\mathrm{m}}$ curve is correlated with the degree of crystallinity. Therefore, the degree of crystallization of the polymer 
membranes could be calculated from the enthalpy of fusion from the DSC measurements, using the following equation:

$$
X_{c}=\frac{\Delta H_{m}}{\Delta H_{m}^{\phi}} \times 100 \%
$$

where $\Delta H_{m}^{\phi}$ is the enthalpy of fusion for pure PVDF, $104.7 \mathrm{~J} \mathrm{~g}^{-1}$ [44], and $\Delta H_{m}$ is the enthalpy of fusion of the PVDF/PMMA blended membrane. The data for $T_{\mathrm{m}}, \Delta H_{m}$, and the degree of crystallization, $X_{\mathrm{c}}$, are tabulated in Table 2. It shows that the amount of PMMA has a slight influence on the $X_{\mathrm{c}}$. The crystallinity of the polymer blend membranes is lower than that of pure PVDF, suggesting that the amorphous domain of the membranes was increased as the PMMA in the polymer blend membrane progressively retards the crystallization of PVDF [43, 45]. This facilitated the absorption and swelling of the liquid electrolyte, resulting in high ionic conductivity of the polymer electrolyte [44].

Fig. 5(a) shows the ionic conductivity at room temperature for all the polymer electrolytes. With the addition of PMMA, it can be observed that the ionic conductivity of the polymer blend increases from $5.73 \times 10^{-6} \mathrm{~S} \mathrm{~cm}^{-1}$ to a maximum of $1.21 \times 10^{-3} \mathrm{~S}$ $\mathrm{cm}^{-1}$ as electrolyte uptake increases from $15 \%$ to $204 \%$. According to Saito et al. [46], there are three possible ways that carrier ions could be transferred within the porous polymer electrolyte: (1) through liquid electrolyte trapped in pores, (2) through an amorphous domain that is swelled by liquid electrolyte, and (3) along molecular chains in the polymer. Since the carrier ion movements along the molecular chains in the polymer is much slower, the increase in ionic conductivity could be attributed to the large amount of trapped liquid electrolyte in the pores that then penetrates into the polymer chains to 
swell the amorphous domains [23, 44, 46, 47]. The temperature dependence of the ionic conductivity for the polymer electrolyte is presented in Fig. 5(b). Typical behavior for a polymer electrolyte was observed, that is, ionic conductivity increases with increasing temperature. Higher temperature not only promotes the migration of carrier ions, but also results in the expansion of polymer [47]. This expansion produces local empty space and increases the free volume, which promotes the motion of polymer segments and carrier ions. The conductivity-temperature plot has a regression value, $R^{2} \geq 0.99$, indicating that the ionic conductivity follows the Arrhenius equation, which is shown in Eq. (5). $\sigma=\sigma_{o} \exp \left(\frac{-E_{a}}{R T}\right)$

where $\sigma$ is the conductivity of the polymer electrolyte, $\sigma_{o}$ is a pre-exponential factor, $E_{a}$ is the activation energy, $R$ is the gas constant, and $T$ is the temperature. From the gradient of the Arrhenius plot, the activation energy, $E_{a}$, for the polymer electrolytes was calculated (Fig. 5(c)). The results suggest that the polymer electrolyte with the highest ionic conductivity has the lowest activation energy. Therefore, the migration of lithium ions is much easier with more tunnels in the polymer membranes [48].

A $\mathrm{Li} / \mathrm{E} 70 / \mathrm{LiFePO}_{4}$ coin-type cell was fabricated and subjected to cycle testing at room temperature. The voltage range was 2.5 to $4.2 \mathrm{~V}$ at a current density of $8.5 \mathrm{~mA} \mathrm{~g}^{-1}$ (corresponding to a C/20 rate). Sample E70 was chosen as the electrolyte, lithium metal as the anode, and $\mathrm{LiFePO}_{4}$ as the cathode. The cell operates on the two-phase reversible process:

$\mathrm{Li}+\mathrm{LiFePO}_{4} \leftrightarrow 2 \mathrm{Li}+\mathrm{FePO}_{4}$ 
with a theoretical specific capacity of $170 \mathrm{mAh} \mathrm{g}^{-1}[49,50]$. The specific capacity versus cycle number of the cell is presented in Fig. 6(a). The charge capacity was higher than the discharge capacity in the first cycle. This irreversible capacity was considered to be due to the irreversible capacity loss related to the formation of the passivation layer at the lithium anode [51, 52]. The charge and discharge capacities of the cell are 150 and 129 $\mathrm{mAh} \mathrm{g}^{-1}$ at the first cycle. After the second cycle, the capacity increases to a maximum at 15 cycles, and then the cell capacity remains at about $135 \mathrm{mAh} \mathrm{g}^{-1}$ up to 50 cycles. The capacity is stable, and the coulombic efficiency approaches $100 \%$ over the 50 cycles. The charge-discharge profiles of the $\mathrm{Li} / \mathrm{E} 70 / \mathrm{LiFePO}_{4}$ cell at the $1^{\text {st }}, 2^{\text {nd }}, 5^{\text {th }}, 10^{\text {th }}, 20^{\text {th }}, 30^{\text {th }}$, $40^{\text {th }}$, and $50^{\text {th }}$ cycles are shown in Fig. 6(b). All charge-discharge curves exhibit a flat plateau at 3.4 and $3.5 \mathrm{~V}$, which is a typical characteristic of $\mathrm{LiFePO}_{4}$. Linear sweep polarization was performed to investigate the electrochemical stability window of Sample E70. For comparison, current-voltage measurement for commercial liquid electrolyte composed of $1 \mathrm{M} \mathrm{LiPF}_{6}$ in EC:DMC (1:1 by volume) was carried out. As shown in Fig. 6(c), it was found that the polymer electrolyte is stable up to $4.7 \mathrm{~V}$, which meets the requirement for a practical lithium-ion battery. This is consistent with other reported gelled polymer electrolytes, since the electrochemical oxidation potential is mainly due to the absorbed liquid electrolyte [3, 22, 47, 48, 53]. Further research is necessery to improve the high rate performance of the lithium-ion batteries. It is well-known that the lithium-polymer battery performs better at high temperature. Therefore, ways to improve the capacity may consist in operating at high temperature. Another strategy to achieve high capacity of lithium-polymer battery is by soaking the porous membrane with highly conductive electrolyte solution. 


\section{Conclusions}

In summary, PVDF/PMMA blend membranes have been successfully prepared via the phase separation method, followed by immersion in commercial liquid electrolyte to activate the membrane. The conformation of the PVDF/PMMA membranes was verified through attenuated total reflectance-Fourier transformation infrared spectroscopy (ATR-FTIR). The degree of crystallinity of a polymer blend membrane decreases as the PMMA concentration increases. The addition of PMMA to the PVDF membrane not only increases the porosity, but also increases the liquid electrolyte uptake. SEM observations showed that Sample M70 has a large pore size $(\sim 1 \mu \mathrm{m})$ when compared with the pure PVDF and the pure PMMA membranes. The ionic conductivity at room temperature of Sample E70 was $1.21 \times 10^{-3} \mathrm{~S} \mathrm{~cm}^{-1}$, and this sample has the lowest activation energy of $16.68 \mathrm{~kJ} \mathrm{~mol}^{-1}$. The $\mathrm{Li} / \mathrm{E} 70 / \mathrm{LiFePO}_{4}$ cell showed stable electrochemical performance up to 50 cycles and an electrochemical stability window that extended up to $4.7 \mathrm{~V}$. This research suggests that PVDF/PMMA polymer electrolyte could be suitable for use as electrolyte for lithium-ion rechargeable batteries.

\section{Acknowledgements}

Financial support provided by the Australian Research Council (ARC) through a Discovery Project (DP 0987805) and ARC Centre of Excellence funding are gratefully acknowledged. Nurul Hayati Idris is grateful to the Ministry of Higher Education of the Government of Malaysia for scholarship support. The authors would like to thank Dr. Tania Silver for critical reading and correction of this manuscript.

\section{References}


[1] A. Manuel Stephan, Eur. Polym. J. 42 (2006) 21.

[2] H.S. Kim, P. Periasamy, S.I. Moon, J. Power Sources 141 (2005) 293.

[3] H.P. Zhang, P. Zhang, Z.H. Li, M. Sun, Y.P. Wu, H.Q. Wu, Electrochem. Commun. 9 (2007) 1700.

[4] Y.P. Wu, H.P. Zhang, F. Wu, Z.H. Li, Polymer Lithium-Ion Batteries, Chemical Industry Press, Beijing, 2007.

[5] F.M. Gray, Solid Polymer Electrolytes-Fundamentals and Technological Applications, VCH, New York, 1991.

[6] B. Scrosati, Applications of Electroactive Polymers, Chapman Hall, London, 1993.

[7] F.M. Gray, Polymer Electrolytes, The Royal Society of Chemistry, Cambridge, 1997.

[8] J.R. MacCallum, Polymer Electrolytes Reviews-I, Elsevier, London, 1987.

[9] J.R. MacCallum, Polymer Electrolytes Reviews-II, Elsevier, London, 1987.

[10] D.E. Fenton, J.M. Parker, P.V. Wright, Polymer 14 (1973) 589.

[11] X.J. Wang, J.J. Kang, Y.P. Wu, S.B. Fang, Electrochem. Commun. 5 (2003) 1025.

[12] L.-Z. Fan, J. Maier, Electrochem. Commun. 8 (2006) 1753.

[13] F. Croce, L. Settimi, B. Scrosati, Electrochem. Commun. 8 (2006) 364.

[14] J.J. Xu, H. Ye, Electrochem. Commun. 7 (2005) 829.

[15] M. Oliver, US Patent 5658685 (1997).

[16] G. Li, Z. Li, P. Zhang, H. Zhang, Y. Wu, Pure Appl. Chem. 80 (2008) 2553.

[17] Q. Shi, M. Yu, X. Zhou, Y. Yan, C. Wan, J. Power Sources 103 (2002) 286.

[18] R.E. Kesting, in: D.R. Lloyd (Ed.) Materials Science of Synthetic Membrane, ACS Symposium Series No. 269, American Chemical Society, Washington, D.C, 1985, pp. 131. 
[19] X.X. Zhu, K. Banana, H.Y. Liu, M. Krause, M. Yang, Macromolecules 32 (1999) 277.

[20] A. Subramania, N.T. Kalyana Sundaram, A.R. Sathiya Priya, G. Vijaya Kumar, J. Membr. Sci. 294 (2007) 8.

[21] J.M. Tarascon, A.S. Gozdz, C. Schmutz, F. Shokoohi, P.C. Warren, Solid State Ionics 86-88 (1996) 49.

[22] G.C. Li, P. Zhang, H.P. Zhang, L.C. Yang, Y.P. Wu, Electrochem. Commun. 10 (2008) 1883.

[23] Z. Li, G. Su, X. Wang, D. Gao, Solid State Ionics 176 (2005) 1903.

[24] J.Y. Song, Y.Y. Wang, C.C. Wan, J. Power Sources 77 (1999) 183.

[25] A. Du Pasquier, P.C. Warren, D. Culver, A.S. Gozdz, G.G. Amatucci, J.M. Tarascon, Solid State Ionics 135 (2000) 249.

[26] M. Watanabe, M. Itoh, K. Sanui, N. Ogata, Macromolecules 20 (1987) 569.

[27] M. Mucha, React. Funct. Polym. 38 (1998) 19.

[28] J.A. Galloway, K.J. Koester, B.J. Paasch, C.W. Macosko, Polymer 45 (2004) 423.

[29] P.A.R.D. Jayathilaka, M.A.K.L. Dissanayake, I. Albinsson, B.E. Mellander, Solid State Ionics 156 (2003) 179.

[30] A. Martinelli, M.A. Navarra, A. Matic, S. Panero, P. Jacobsson, L. Börjesson, B. Scrosati, Electrochim. Acta 50 (2005) 3992.

[31] S. Ramesh, A.K. Arof, J. Power Sources 99 (2001) 41.

[32] N.-S. Choi, J.-K. Park, Electrochim. Acta 46 (2001) 1453.

[33] G. Girish Kumar, S. Sampath, Solid State Ionics 176 (2005) 773.

[34] S. Ahmad, S. Ahmad, S.A. Agnihotry, J. Power Sources 140 (2005) 151. 
[35] D. Saikia, A. Kumar, Eur. Polym. J. 41 (2005) 563.

[36] Y. Saito, A.M. Stephan, H. Kataoka, Solid State Ionics 160 (2003) 149.

[37] Z.H. Li, P. Zhang, H.P. Zhang, Y.P. Wu, X.D. Zhou, Electrochem. Commun. 10 (2008) 791.

[38] J. Xi, X. Qiu, J. Li, X. Tang, W. Zhu, L. Chen, J. Power Sources 157 (2006) 501.

[39] B. Oh, Y.R. Kim, Solid State Ionics 124 (1999) 83.

[40] S. Rajabzadeh, T. Maruyama, Y. Ohmukai, T. Sotani, H. Matsuyama, Sep. Purif. Technol. 66 (2009) 76.

[41] I.S. Elashmawi, N.A. Hakeem, Polym. Eng. Sci. 48 (2008) 895.

[42] Z. Li, J. Wei, F. Shan, J. Yang, X. Wang, J. Polym. Sci., Part B: Polym. Phys. 46 (2008) 751.

[43] W. Ma, J. Zhang, X. Wang, S. Wang, Appl. Surf. Sci. 253 (2007) 8377.

[44] J.-H. Cao, B.-K. Zhu, Y.-Y. Xu, J. Membr. Sci. 281 (2006) 446.

[45] J. R. Gregorio, N.C.P.d.S. Nociti, J. Phys. D: Appl. Phys. 28 (1995) 432.

[46] Y. Saito, H. Kataoka, E. Quartarone, P. Mustarelli, J. Phys. Chem. B 106 (2002) 7200.

[47] Z.-Y. Cui, Y.-Y. Xu, L.-P. Zhu, J.-Y. Wang, Z.-Y. Xi, B.-K. Zhu, J. Membr. Sci. 325 (2008) 957.

[48] Z. Li, G. Su, D. Gao, X. Wang, X. Li, Electrochim. Acta 49 (2004) 4633.

[49] V. Gentili, S. Panero, P. Reale, B. Scrosati, J. Power Sources 170 (2007) 185.

[50] A.K. Padhi, K.S. Nanjundaswamy, C. Masquelier, S. Okada, J.B. Goodenough, J. Electrochem. Soc. 144 (1997) 1609. 
[51] Q. Hu, S. Osswald, R. Daniel, Y. Zhu, S. Wesel, L. Ortiz, D.R. Sadoway, J. Power Sources 196 (2011) 5604.

[52] E. Peled, D. Golodnitsky, G. Ardel, J. Electrochem. Soc. 144 (1997) L208.

[53] P. Zhang, H.P. Zhang, G.C. Li, Z.H. Li, Y.P. Wu, Electrochem. Commun. 10 (2008) 1052.

\section{Figure captions}

Fig. 1. Cross-sectional SEM images of porous membrane for Sample (a) M0, (b) M30, (c) M50, (d) M70, (e) M90, and (f) M100.

Fig. 2. Porosity and liquid electrolyte uptake as a function of PMMA weight fraction in blended porous membranes with polymer electrolyte.

Fig. 3. ATR-FTIR spectra of Sample (a) M0, (b) M30, (c) M50, (d) M70, (e) M90, and (f) M100 from 1000-2000 $\mathrm{cm}^{-1}$. The graph in the inset shows the ATR-FTIR spectra of Sample M30, M50, M70, and M100 in the range of 1660 to1800 $\mathrm{cm}^{-1}$.

Fig. 4. DSC curves of pure PVDF, pure PMMA, and polymer blend membranes.

Fig. 5. (a) Plots of ionic conductivity as a function of PMMA weight fraction in the polymer membranes at room temperature, (b) Arrhenius plots of the ionic conductivities of the polymer electrolytes, and (c) activation energy of polymer electrolytes.

Fig. 6. Electrochemical performances of $\mathrm{Li} / \mathrm{E} 70 / \mathrm{LiFePO}_{4}$ cells: (a) cyclic performance and coulombic efficiency beyond 50 cycles at $8.5 \mathrm{~mA} \mathrm{~g}^{-1}$ (corresponding to a $\mathrm{C} / 20$ rate); (b) charge-discharge profiles for selected cycles; (c) linear sweep voltammograms of Sample E70 and commercial liquid electrolyte with a stainless steel working electrode and a lithium metal counter electrode at a scan rate of $1 \mathrm{mV} \mathrm{s}^{-1}$. 


\section{Table captions}

Table 1. Compositions of PVDF/PMMA membranes and corresponding polymer electrolytes.

Table 2. Degree of crystallization of PVDF/PMMA blended membranes. 